\title{
Formas utilizadas para tentativa de suicídio e características sociodemográficas dos pacientes atendidos no serviço de emergência de um hospital de ensino
}

\author{
Methods used in suicide attempts and sociodemographic characteristics \\ of patients treated at the emergency department of a teaching hospital
}

\author{
Betina Brixner ${ }^{1} \bowtie$, Caroline Lau Koch ${ }^{1}$, Marla Pedroso Marth${ }^{1}$, Alice Pereira Freitas ${ }^{1}$, Cristiane Carla Dressler Garske ${ }^{1}$, \\ Vanessa Monigueli Giehl'1, Ana Paula Helfer Schneider ${ }^{2}$ \\ Programa de Pós-Graduação em Residência Multiprofissional Integrada em Saúde, concentração em Urgência, Emergência e Intensivismo. \\ Universidade de Santa Cruz do Sul (UNISC). Santa Cruz do Sul, RS. \\ ${ }^{2}$ Curso de Pós-Graduação em Prescrição Farmacêutica e Farmácia Clínica da UNISC; Programa de Pós-Graduação em Saúde Coletiva da \\ Universidade do Vale do Rio dos Sinos (UNISINOS). São Leopoldo, RS.
}

\section{RESUMO}

Objetivos: Caracterizar as formas utilizadas para tentativa de suicídio e características sociodemográficas de pacientes atendidos no serviço de emergência de um hospital de ensino.

Métodos: Estudo transversal retrospectivo, com busca ativa em prontuários eletrônicos de pacientes atendidos entre janeiro e dezembro de 2015 no serviço de emergência de um hospital de ensino, que integra a rede pública de saúde do interior do estado do Rio Grande do Sul, Brasil. Foram incluídos todos os casos de tentativa de suicídio, independente de sua forma. Não foram investigados os casos que resultaram em óbito por suicídio. Foi utilizada estatística descritiva e foram avaliadas associações por análise univariada, sendo aplicado o teste do quiquadrado de Pearson.

Resultados: No período estudado foram encaminhados 233 pacientes atendidos na emergência para realização de avaliação psiquiátrica, sendo 122 casos caracterizados por tentativa de suicídio. Desses, a média de idade dos pacientes foi de 36,8土14,3 anos, com mínima de 14 e máxima de 88 anos e, $74(60,6 \%)$ eram do sexo feminino. As formas mais utilizadas de tentativa de suicídio foram ingesta de medicamentos (72 casos, 59\%) e ingesta de agrotóxicos (14 casos, 11,5\%). Do total de pacientes, $119(97,5 \%)$ residiam no município de Santa Cruz do Sul, com predominância nos moradores da zona urbana (103 pacientes, 84,4\%). Dos $50(41,0 \%)$ encaminhamentos, $32(64,0 \%)$ foram para o Centro de Atendimento Psicossocial, 7 (14\%) para Centro de Atendimento Psicossocial da Infância e Adolescência, 6 (12\%) para internação em unidade de saúde mental e $5(10 \%)$ para o Centro de Atendimento Psicossocial Álcool e Drogas. Em 66 (54,1\%) pacientes não foi encontrado nenhum registro em prontuário eletrônico sobre o desfecho após a alta e $6(4,9 \%)$ pacientes evadiram.

Conclusões: A maioria dos pacientes atendidos por tentativa de suicídio foram adultos jovens do sexo feminino. Como principal forma utilizada para a tentativa de suicídio identificou-se a ingesta de medicamentos e em seguida a de agrotóxicos.

DESCRITORES: tentativa de suicídio; serviços médicos de emergência; substâncias tóxicas; epidemiologia.

\section{ABSTRACT}

Aims: To describe the methods used in suicide attempts and sociodemographic characteristics of suicidal patients treated at the emergency department of a teaching hospital.

Methods: Observational and descriptive study, with active search for electronic medical records of patients treated at the emergency department of a public teaching hospital in the countryside of Rio Grande do Sul, Brazil, from January to December 2015. All cases of suicide attempt were included, regardless of the suicide methods used. Successful suicide cases were not investigated. The statistical analyses included descriptive statistics, univariate analysis, and Pearson's chi-square test.

Results: A total of 233 patients treated at the emergency department were referred for psychiatric evaluation, and 122 of them had attempted suicide. The mean age of the suicidal patients was 36.8 14.3 years, (minimum of 14 years and maximum of 88 years) and $74(60.6 \%)$ of them were female. The most common suicide attempt methods were ingestion of medications ( 72 cases, $59 \%)$ and of pesticide (14 cases, $11.5 \%)$ Of all patients, 119 (97.5\%) lived in Santa Cruz do Sul, predominantly in the urban area (103 patients, 84.4\%). Of the 50 (41.0\%) referrals, $32(64.0 \%)$ were sent to the Center for Psychosocial Care, 7 (14\%) to the Center for Children's and Adolescents' Psychosocial Care, $6(12 \%)$ were admitted to a mental health unit, and $5(10 \%)$ were sent to Psychosocial Care Center for Alcohol and Drug Abuse. Sixty-six (54.1\%) patients had no electronic medical records of their outcome after discharge and 6 (4.9\%) were lost to follow-up.

Conclusions: Most of the treated suicidal patients were young adult females. The most common method of suicide attempt was the ingestion of medications, followed by the ingestion of pesticides.

KEY WORDS: suicide, attempted; emergency medical services; toxic substances; epidemiology.

\section{INTRODUÇÃO}


Abreviatura: CAPS, Centro de Atendimento Psicossocial.

O suicídio constitui uma importante questão de saúde pública no mundo inteiro, sendo conceituado pela Organização Mundial de Saúde como um ato intencional de um indivíduo para extinguir sua própria vida [1]. Já o comportamento suicida pode ser compreendido como "todo ato pelo qual um indivíduo causa lesão a si mesmo, independente do grau de intenção letal e do verdadeiro motivo desse ato" [2]. Estima-se que, até 2020, mais de 1,5 milhão de pessoas poderão cometer suicídio [3].

Considera-se que existem inúmeros fatores associados ao aumento dos índices de suicídio, dentre os quais destaca-se a relação com as doenças e transtornos mentais, como a depressão, abuso/ dependência de álcool e drogas, histórico de tentativas prévias e de suicídio na família, eventos estressores, além dos determinantes e condicionantes da saúde, tais como trabalho, renda e acesso aos bens e serviços [4].

Tendo em vista a extrema relevância da temática, no ano de 2006 o Ministério da Saúde instituiu as Diretrizes Nacionais para Prevenção do Suicídio. Entre os principais objetivos a serem alcançados por esse programa, destacam-se o fomento e a execução de projetos estratégicos fundamentados em estudos de eficácia e qualidade; processos de organização da rede de atenção e intervenções nos casos de tentativas de suicídio; e educação permanente dos profissionais de saúde da atenção básica, inclusive do Programa Saúde da Família, dos serviços de saúde mental e das unidades de urgência e emergência [5].

$\mathrm{Na}$ última década, o Brasil vem registrando um aumento significativo no coeficiente de mortes por suicídio. De acordo com o Mapa de Violência do Ministério da Saúde, o índice nacional de jovens que tiraram a própria vida aumentou $40 \%$ em 10 anos. O Rio Grande do Sul aparece em primeiro lugar nos índices nacionais, uma vez que engloba 11 das 20 cidades brasileiras que mais tiveram casos registrados de suicídio, sendo Santa Cruz do Sul a 11 1 a colocada no país $[6,7]$.

Diante dessa realidade, o presente estudo teve por objetivo caracterizar os aspectos sociodemográficos e clínicos dos casos de tentativa de suicídio atendidos em um serviço de emergência pelo Sistema Único de Saúde, num hospital do interior do Rio Grande do Sul, Brasil.

\section{MÉTODOS}

Este estudo apresenta um delineamento transversal retrospectivo, cuja população alvo constituiu-se de pacientes atendidos por tentativa de suicídio, no serviço de emergência vinculado a uma entidade hospitalar filantrópica e de ensino, situada na cidade de Santa Cruz do Sul, no interior do Rio Grande do Sul, sendo o principal centro de saúde da região. Salienta-se que esse serviço de saúde não é o único que atende casos de urgência e emergência no município. Foram incluídos todos os casos de tentativa de suicídio atendidos pelo Sistema Único de Saúde, independentemente de sua forma. Casos que resultaram em óbito por suicídio não foram investigados.

Para coleta de dados foi realizada busca ativa em prontuários eletrônicos de 233 pacientes que foram triados para avaliação psiquiátrica no referido serviço, no período de janeiro a dezembro de 2015 . O levantamento e a análise dos dados foram realizados no primeiro semestre de 2016. O desfecho forma de tentativa de suicídio foi avaliado de forma dicotômica para cada uma das formas de tentativa de suicídio (medicamento, agrotóxico, enforcamento, objeto cortante, via pública, queda de altura e arma de fogo). As variáveis independentes avaliadas foram: período (janeiro a dezembro de 2015), sexo e idade do paciente ( $<20$ anos, 21-39 anos, 40-59 anos, 60-79 anos e >79 anos), município de Santa Cruz do Sul ou outro, microárea de residência em Santa Cruz do Sul (Zona Sul, Zona Norte, Zona Rural) e encaminhamentos para outros serviços da rede pública de saúde: Centro de Atendimento Psicossocial (CAPS), CAPS Infância e Adolescência, CAPS Álcool e Drogas e internação em unidade de saúde mental.

Os dados foram digitados no programa Microsoft Excel $^{\circledR} 2013$ e analisados no programa IBM SPSS Statistics versão 20.0. Foram realizadas análises descritivas e análises de associação univariadas, sendo aplicados os testes qui-quadrado de Pearson e associação linear, considerando estatisticamente significativos os resultados com $\mathrm{p}<0,05$.

O estudo foi previamente autorizado pela instituição hospitalar e aprovado pelo Comitê de Ética em Pesquisa da Universidade de Santa Cruz do Sul em 01 de março de 2016, sob o parecer 1.432.385/16, sendo dispensado termo de consentimento livre e esclarecido por tratar-se de levantamento retrospectivo.

\section{RESULTADOS}

O serviço de emergência estudado registra em média 75,6 mil atendimentos ao ano, sendo que a 
média diária chega a 200 atendimentos. Destes, foram examinados 233 prontuários eletrônicos de pacientes que passaram por avaliação psiquiátrica, sendo que 111 não correspondiam aos critérios de inclusão, sendo, portanto incluídos 122 pacientes no estudo.

Durante o ano de 2015, o mês de março foi o que apresentou maior ocorrência de tentativas de suicídio, com 15 (12,5\%) casos, seguido de maio com $14(11,5 \%)$ e abril com $13(10,7 \%)$ casos. Dos 122 pacientes avaliados por tentativas de suicídio, a média de idade apresentada foi de $36,8 \pm 14,3$ anos, com idade mínima de 14 e máxima de 88 anos, e 74 (60,6\%) eram pacientes do sexo feminino. Observou-se que a forma de tentativa de suicídio mais frequente ocorreu por ingesta de medicamentos, 72 casos $(59,0 \%)$, seguida por ingesta de agrotóxicos, com 14 casos $(11,5 \%)$.

Referente às formas de tentativa de suicídio em relação à faixa etária dos pacientes, nota-se que 57 $(46,7 \%)$ casos de tentativas ocorreram em pacientes entre 21-39 anos. Os casos de ingesta de medicamento só não predominaram como forma de tentativa de suicídio nos idosos (60 anos ou mais) sendo a mais utilizada em todas as outras faixas etárias. As associações estatisticamente significativas em relação à faixa etária ocorreram entre a faixa 60-79 anos e a tentativa de suicídio por atropelamento autoprovocado em via pública, e entre as faixas mais jovens ( $<20$ anos) e mais velhas ( $>79$ anos) com a tentativa de suicídio por enforcamento (Tabela 1).

Já em relação a formas de tentativa de suicídio e sexo dos pacientes, pode-se observar que o uso de medicação como forma de tentativa de suicídio foi ligeiramente superior nas mulheres $(63,5 \%$ dos casos versus todas as outras formas) em comparação aos homens $(52,1 \%$ dos casos versus todas as outras formas). Entretanto não foi encontrada nenhuma associação estatisticamente significativa entre o sexo e a forma de tentativa de suicídio (Tabela 2).

Tabela 1. Relação das formas de tentativa de suicídio com a faixa etária dos pacientes atendidos no serviço de emergência de um hospital de ensino, que integra a rede pública de saúde do interior do Rio Grande do Sul, Brasil, no ano de 2015.

\begin{tabular}{|c|c|c|c|c|c|c|c|}
\hline \multirow[b]{2}{*}{ Formas de tentativa* } & \multicolumn{6}{|c|}{ Faixa etária (anos) } & \multirow[b]{2}{*}{$\mathbf{p}^{*}$} \\
\hline & $\begin{array}{l}<20 \\
\text { n }(\%)\end{array}$ & $\begin{array}{c}21-39 \\
\text { n (\%) }\end{array}$ & $\begin{array}{l}40-59 \\
n(\%)\end{array}$ & $\begin{array}{l}60-79 \\
n(\%)\end{array}$ & $\begin{array}{l}>79 \\
\text { n (\%) }\end{array}$ & $\begin{array}{l}\text { Total } \\
\text { n (\%) }\end{array}$ & \\
\hline Medicamentos & $12(85,7)$ & $33(57,9)$ & $26(57,8)$ & $1(20,0)$ & - & $72(59,0)$ & 0,065 \\
\hline Agrotóxicos & - & $9(15,8)$ & $5(11,1)$ & - & - & $14(11,5)$ & 0,448 \\
\hline Enforcamento & $2(14,3)$ & $5(8,8)$ & $1(2,2)$ & $1(20,0)$ & $1(100,0)$ & $10(8,2)$ & 0,007 \\
\hline Objeto cortante & - & $4(7,0)$ & $5(11,1)$ & $1(20,0)$ & - & $10(8,2)$ & 0,553 \\
\hline Via pública** & - & $3(5,3)$ & - & $2(40,0)$ & - & $5(4,1)$ & 0,001 \\
\hline Queda de altura & - & - & $4(8,9)$ & - & - & $4(3,3)$ & 0,108 \\
\hline Arma de fogo & - & $1(1,8)$ & - & - & - & $1(0,8)$ & 0,891 \\
\hline Não consta & - & $2(3,5)$ & $4(8,9)$ & - & - & $6(4,9)$ & - \\
\hline Total & $14(100,0)$ & $57(100,0)$ & $45(100,0)$ & $5(100,0)$ & $1(100,0)$ & $122(100,0)$ & \\
\hline
\end{tabular}

* Teste do Qui-quadrado para associação linear; ** Atropelamento autoprovocado.

Tabela 2. Associações entre formas de tentativa de suicídio e sexo dos pacientes atendidos no serviço de emergência de um hospital de ensino, que integra a rede pública de saúde do interior do Rio Grande do Sul, Brasil, no ano de 2015

\begin{tabular}{lcccc}
\hline \multicolumn{1}{c}{ Formas de tentativa } & $\begin{array}{c}\text { Masculino } \\
\mathbf{n}(\%)\end{array}$ & $\begin{array}{c}\text { Feminino } \\
\mathbf{n}(\%)\end{array}$ & $\begin{array}{c}\text { Total } \\
\mathbf{n}(\%)\end{array}$ & p $^{*}$ \\
\cline { 2 - 3 } Medicamentos & $25(52,1)$ & $47(63,5)$ & $72(59,0)$ & 0,104 \\
\hline Agrotóxicos & $6(12,5)$ & $8(10,8)$ & $14(11,5)$ & 0,849 \\
\hline Enforcamento & $5(10,4)$ & $5(6,8)$ & $10(8,2)$ & 0,523 \\
\hline Objeto cortante & $6(12,5)$ & $4(5,4)$ & $10(8,2)$ & 0,189 \\
Via pública** & $2(4,2)$ & $3(4,1)$ & $5(4,1)$ & 0,981 \\
Queda de altura & $2(4,2)$ & $2(2,7)$ & $4(3,3)$ & 0,694 \\
Arma de fogo & $1(2,1)$ & - & $1(0,8)$ & 0,224 \\
\hline Não consta & $1(2,1)$ & $5(6,8)$ & $6(4,9)$ & - \\
\hline Total & $48(100,0)$ & $74(100,0)$ & $122(100,0)$ & \\
\hline
\end{tabular}

* Teste do Qui-quadrado de Person; ** Atropelamento autoprovocado. 
Tabela 3. Associações entre formas de tentativas de suicídio e local de residência dos pacientes atendidos no serviço de emergência de um hospital de ensino, que integra a rede pública de saúde do interior do Rio Grande do Sul, Brasil, no ano de 2015

\begin{tabular}{|c|c|c|c|c|c|c|}
\hline \multirow[b]{2}{*}{ Formas de tentativa } & \multicolumn{3}{|c|}{ Microárea do Município de Santa Cruz do Sul } & \multirow{2}{*}{$\begin{array}{c}\text { Outro Município } \\
\text { n (\%) }\end{array}$} & \multirow{2}{*}{$\begin{array}{l}\text { Total } \\
\text { n (\%) }\end{array}$} & \multirow[b]{2}{*}{$\mathbf{p}^{*}$} \\
\hline & $\begin{array}{c}\text { Zona Sul } \\
\text { n (\%) }\end{array}$ & $\begin{array}{l}\text { Zona Norte } \\
\text { n (\%) }\end{array}$ & $\begin{array}{l}\text { Zona Rural } \\
\text { n (\%) }\end{array}$ & & & \\
\hline Medicamentos & $47(58,8)$ & $13(56,5)$ & $11(68,8)$ & $1(33,3)$ & $72(59,0)$ & 0,632 \\
\hline Agrotóxicos & $7(8,8)$ & $3(13,0)$ & $3(18,8)$ & $1(33,3)$ & $14(11,5)$ & 0,485 \\
\hline Enforcamento & $7(8,8)$ & $3(13,0)$ & - & - & $10(8,2)$ & 0,488 \\
\hline Objeto cortante & $7(8,8)$ & $2(8,7)$ & $1(6,2)$ & - & $10(8,2)$ & 0,927 \\
\hline Via pública** & $3(3,8)$ & $1(4,3)$ & $1(6,2)$ & - & $5(4,1)$ & 0,961 \\
\hline Queda de altura & $3(3,8)$ & $1(4,3)$ & - & - & $4(3,3)$ & 0,846 \\
\hline Arma de fogo & - & - & - & $1(33,3)$ & $1(0,8)$ & $<0,001$ \\
\hline Não consta & $6(7,5)$ & - & - & - & $6(4,9)$ & - \\
\hline Total & $80(100,0)$ & $23(100,0)$ & $16(100,0)$ & $3(100,0)$ & $122(100,0)$ & \\
\hline
\end{tabular}

* Teste do Qui-quadrado de Person; ** Atropelamento autoprovocado.

Dos 122 pacientes do estudo, 119 (97,5\%) eram residentes do município de Santa Cruz do Sul e 3 (2,5\%) de outros municípios. Dos moradores de Santa Cruz do Sul, $103(84,4 \%)$ eram habitantes da zona urbana e $16(13,4 \%)$ da zona rural. Ao confrontar as formas de tentativa de suicídio com o local de residência, verificou-se que houve somente uma tentativa por arma de fogo, e esse paciente era um dos três residentes em outro município que não Santa Cruz do Sul. Sendo assim, a associação entre uso de arma de fogo e local de residência foi estatisticamente significativa, enquanto nenhuma outra associação foi identificada entre local de residência e forma de tentativa de suicídio (Tabela 3).

Quanto ao desfecho dos pacientes, em 66 (54,1\%) casos não houve registro em prontuário eletrônico e $6(4,9 \%)$ dos pacientes evadiram. Dos $50(41,0 \%)$ encaminhamentos, $32(64,0 \%)$ foram para o CAPS II, 7 (14\%) para o CAPS da Infância e Adolescência, seis (12\%) para internação em unidade de saúde mental e cinco $(10 \%)$ para o CAPS Álcool e Drogas.

\section{DISCUSSÃO}

Apesar de não haver registros das tentativas de suicídio a nível mundial, estima-se que essas taxas chegam a ser pelo menos 10 vezes maiores do que os registros de suicídio $[8,9]$. O grande número dessas ocorrências pôde ser identificado no presente estudo, o qual demonstrou que a cada três dias ocorreu uma tentativa de suicídio. Estima-se que o número possa ser muito maior, pois um estudo realizado em Campinas verificou que de cada três pessoas que tentaram se suicidar, apenas uma foi atendida em prontosocorro [10].

Os atendimentos por tentativas de suicídio neste estudo ocorreram predominantemente no mês de março $(12,3 \%)$, resultado próximo ao encontrado em outro estudo realizado em pronto socorro na cidade de Taubaté, SP, que descreve maior incidência em fevereiro, com $51(12,63 \%)$ tentativas de suicídio [11]. Em ambos os estudos, as maiores incidências foram encontradas no verão, e há estudos $[12,13]$ que sugerem diferenças no comportamento suicida de acordo com as variações climáticas. No caso do verão, há relação entre a luz do sol, altas temperaturas e taxa de suicídio. Além disso, Petridou et al. [13] considera que o elevado risco de suicídio durante os meses de verão, pode estar associado a mudanças de comportamento das pessoas, durante o período de férias de trabalho e com mais tempo livre.

No que se refere ao sexo, neste estudo $60,6 \%$ das tentativas de suicídio foram realizadas por mulheres, mas a diferença não foi significativa em relação ao sexo masculino. Outros estudos, contudo, encontraram que as mulheres são as que mais tentam suicídio, porém utilizam, na maioria dos casos, métodos menos efetivos, diferentemente dos casos de óbito por suicídio, nos quais o sexo masculino é predominante. Alguns estudos observaram predominância de meios letais no sexo masculino [14-18]. No presente estudo, embora não tenham sido encontradas diferenças significativas, foi possível visualizar uma tendência ao predomínio das formas potencialmente mais letais (enforcamento, objeto cortante e arma de fogo) nos indivíduos do sexo masculino. 
Este estudo identificou a faixa etária de adulto jovem (20 a 39 anos) como a de maior risco de tentar suicídio. Este fato pode estar associado a aspectos de insegurança e estresse no emprego, desemprego, crise econômica, bem como vínculos sociais e familiares fragilizados, que podem contribuir para o adoecimento psíquico [19,20].

A forma de tentativa de suicídio mais frequente, em ambos os sexos, foi a intoxicação exógena. A intoxicação exógena chega a ser responsável por aproximadamente $70 \%$ dos casos notificados no Brasil [21]. Os resultados encontrados mostram que em $59 \%$ dos casos de intoxicação exógena foram utilizados medicamentos, enquanto $11,5 \%$ ocorreram por meio da ingesta de agrotóxicos. Outro estudo destaca que os agentes tóxicos mais comumente relacionados à tentativa de suicídio são os medicamentos ansiolíticos e antidepressivos, demostrando que os pacientes com quadro de violência autodirigida já apresentam algum tipo de histórico referente à instabilidade emocional [15].

Evidenciou-se uma possível facilidade de acesso a esses produtos farmacêuticos, talvez contribuindo para uma maior ocorrência de tentativas de suicídio por intoxicação. Sendo assim, enfatiza-se a importância de atentar para fatores como a distribuição e a comercialização de fármacos, prescrição indiscriminada de medicações e acesso a substâncias tóxicas dirigidas para outras finalidades e adquiridas livremente sem regulamentação [14].

Em relação às microáreas municipais incluídas neste estudo, houve predominância das tentativas de suicídio nos moradores de áreas urbanas, principalmente da zona sul; situação semelhante à encontrada em uma cidade na Colômbia, no qual $73 \%$ dos adolescentes que cometeram suicídio residiam em área urbana [23]. Entretanto, não foi realizada uma pesquisa abrangente de forma a identificar se as pessoas residentes em áreas rurais poderiam ter sido atendidas em outros locais. Outros estudos encontraram situação diferente, com predominância das tentativas de suicídio em moradores das áreas rurais. Esses autores conjeturam que os cuidados de saúde em zonas rurais é baixo e que a maioria das pessoas não possui acesso a cuidados de saúde primários, principalmente aos serviços de saúde mental, o que poderia atuar como fator de risco para a tendência suicida [14,24,25].

Em relação aos encaminhamentos realizados após o atendimento no serviço de emergência, em mais da metade dos prontuários eletrônicos examinados não foram encontrados registros de encaminhamento para outro serviço de saúde do município. Ressalta-se que a Cartilha Nacional de Prevenção ao Suicídio de 2014 aponta que grande parte das mortes por suicídio nunca passaram pela rede de saúde mental, sendo que em torno de $50 \%$ dos pacientes procuram médicos generalistas seis meses antes de cometer o ato suicida e em torno de $80 \%$ um mês antes [20]. Sendo assim, sugere-se a implantação de um protocolo específico para identificação de possíveis comportamentos suicidas. É necessário qualificar o serviço prestado, fortalecer a rede de atenção psicossocial e subsidiar outros estudos com vistas à elaboração e implantação de projetos e programas tendo esta temática como alvo.

Ressalta-se que este estudo limitou-se às tentativas de suicídio atendidas no serviço de emergência de um hospital, não evidenciando a realidade de outros serviços de saúde no município de Santa Cruz do Sul. Outra limitação deste estudo foi a coleta de dados somente nos prontuários eletrônicos, não havendo busca ativa nos prontuários físicos de cada paciente para averiguar se havia outras informações descritas como, por exemplo, encaminhamentos para outros serviços de atenção básica ou especializados.

Concluímos que dos pacientes atendidos por tentativa de suicídio no serviço de emergência estudado, a predominância foi de pacientes do sexo feminino, com destaque para a faixa etária entre 21 e 39 anos, moradores da zona urbana. A forma mais utilizada para a tentativa de suicídio foi a ingesta de medicamentos, seguida pela de agrotóxicos. Na maioria dos casos não constava no prontuário eletrônico o encaminhamento realizado para outro serviço de saúde, salientando desta forma, a importância de reavaliar a rotina de registro dos encaminhamentos efetuados nos prontuários eletrônicos do serviço estudado. 
NOTA

\section{Declaração de conflitos de interesse}

Os autores declaram não haver conflitos de interesse relevantes ao conteúdo deste estudo.

\section{REFERÊNCIAS}

1. World Health Organization. World report on violence and health. Geneva: WHO; 2002.

2. Botega NJ. Crise Suicida: avaliação e manejo. Porto Alegre: Artmed; 2015.

3. Bertolote JM, Fleischmann A. A global perspective in the epidemiology of suicide. Suicidology. 2002;7(2):6-8

4. Skogman K, Alsén M, Öjehagen A. Sex differences in risk factors for suicide after attempted suicide: a follow-up study of 1052 suicide attempters. Soc Psychiatry Pschiatr Epidemiol. 2004;39(2):113-20. http://dx.doi.org/10.1007/s00127-004-0709-9

5. Ministério da Saúde (BR). Secretaria de Atenção à Saúde. Departamento de Ações Programáticas Estratégicas. Área Técnica de Saúde Mental. Prevenção do suicídio: manual dirigido a profissionais das equipes de saúde mental. Brasília: MS; 2006.

6. Waiselfisz JJ. Mapa da Violência. Homicídios e Juventude no Brasil. Secretaria Nacional de Juventude. Brasília; 2013.

7. Governo do Estado do Rio Grande do Sul. Fundação de Economia e Estatística. Perfil Socioeconômico do município de Santa Cruz do Sul [Internet]. Porto Alegre: FEE; 2016 [cited 2016 Mar 15]. Available from: http://www.fee.rs.gov.br/perfilsocioeconomico/municipios/ detalhe/?municipio=Santa + Cruz + do + Sul

8. World Health Organization. Preventing suicide: a global imperative. Geneva: WHO; 2014.

9. Botega NJ. Comportamento suicida: epidemiologia. Psicologia USP. 2014;25(3):231-6. http://dx.doi.org/10.1590/0103-6564D20140004

10. Botega NJ, Marín-León L, Oliveira HB, Barros MB, Silva VF, Dalgalarrondo P. Prevalências de ideação, planos e tentativas de suicídio: um inquérito populacional em Campinas SP. Cad Saúde Pública. 2009;25(12):2632-8. http://dx.doi.org/10.1590/S0102-311X2009001200010

11. Lara EM, Xavier MS, Gonçalves M. Perfil dos pacientes envolvidos em tentativas de suicídio por intoxicação exógena no pronto socorro de Taubaté. Psychiatry Online Brasil. 2009 [cited 2016];14(9). Available from: http://www.polbr.med.br/ano09/prat0909.php

12. Kposowa AJ, D'Auria S. Association of temporal factors and suicides in the United States, 2000-2004. Soc Psychiat Epidemiol 2010;45(4):433-45. http://dx.doi.org/10.1007/s00127-009-0082-9

13. Petridou E, Papadopoulos FC, Frangakis CE, Skalkidou A, Trichopoulos D.A Role of Sunshine in the Triggering of Suicide. Epidemiology. 2002;13:106-9 http://dx.doi.org/10.1097/00001648-200201000-00017

14. Moreira DL, Martins MC, Gubert FA, Sousa FSP. Perfil de pacientes atendidos por tentativa de suicídio em um Centro de Assistência. Toxicológica. Cienc Enferm. 2015;20(2):63-75. http://dx.doi.org/10.4067/S0717-95532015000200007

15. Oliveira EN, Félix TA, Mendonça CBL, Souza DR, Ferreira GB, Freire MA, Lima PSF, Teodósio TBT, Linhares JM, Almeida PC. Tentativa de suicídio por intoxicação exógena: contexto de notificações compulsórias Rev Eletrônica Gest Saúde. 2015;6(3):2497-11.

16. Macente LB, Santos EG, Zandonade E. Tentativas de suicídio e suicídio em município de cultura pomerana no interior do estado do Espírito Santo. J Bras Psiquiatr. 2009;58(4):238-44. http://dx.doi.org/10.1590/S0047-20852009000400004

17. Lovisi GM, Santos AS, Legay L, Abelha L, Valencia E. Epidemiological analysis of suicide in Brazil from 1980 to 2006 . Rev Bras Psiquiatr 2009 Oct;31 Suppl 2:S86-94

18. Bertolote JM, Fleischmann, AA global perspective on the magnitude of suicide mortality. In: Wasserman D, Wasserman C, editors Oxford textbook of suicide and suicide prevention. Oxford: Oxford University Press; 2009. p. 91-98. http://dx.doi.org/10.1093/med/ 9780198570059.003 .0014

19. Dalglish SL, Melchior M, Younes N, Surkan PJ. Work characteristics and suicidal ideation in young adults in France. Soc Psychiatry Psychiatr Epidemiol. 2015 Apr;50(4):613-20. http://dx.doi.org/10.1007/s00127-014-0969-y

20. Park SM, Cho SI, Moon SS. Factors associated with suicidal ideation: Role of emotional and instrumental support. J Psychosom Res. 2010 Oct;69(4):389-97. http://dx.doi.org/10.1016/j.jpsychores.2010.03.002

21. Santos SA, Legay LF, Lovisi GM. Substâncias tóxicas e tentativas e suicídios: considerações sobre acesso e medidas restritivas. Cad Saúde Colet. 2013;21(1):53-61. http://dx.doi.org/10.1590/S1414-462X2013000100009

22. Cardoso HF, Baptista MN, Ventura CD, Branão EM, Padovan, FD, Gomes MA. Suicídio no Brasil e América Latina: revisão bibliométrica na base de dados Redalycs. Diaphora. 2012. 12(2): 42-8.

23. Medina-Pérez OA, Díaz-Téllez AS, Rozo-David AJ. Caracterización del suicídio em adolescentes de Antioquia, Colombia, 2000-2010. Rev Fac Med. 2015;63(3):431-8. http://dx.doi.org/10.15446/revfacmed.v63n3.43798

24. Bruffaerts R, Demyttenaere K, Hwang I, Chiu WT, Sampson N, Kessler RC, Alonso J, Borges G, de Girolamo G, de Graaf R, Florescu S, Gureje O, Hu C, Karam EG, Kawakami N, Kostyuchenko S, Kovess-Masfety V, Lee S, Levinson D, Matschinger H, Posada-Villa J, Sagar R, Scott KM, Stein DJ, Tomov T, Viana MC, Nock MK. Treatment of suicidal people around the world. Br J Psychiatry. 2011 July;199(1):64-70. http://dx.doi.org/10.1192/bjp.bp.110.084129

25. Wagenaar BH, Hagaman AK, Kaiser BN, McLean KE, Kohrt BA. Depression, suicidal ideation, and associated factors: a cross-sectional study in rural Haiti. BMC Psychiatry. 2012 Sep 19;12:149. http://dx.doi.org/10.1186/1471-244X-12-149 